\title{
CHARACTERIZATION OF AIRBORNE ASPERGILLUS SERIES VERSICOLORES COLLECTED IN FRENCH BIOAEROSOLS
}

\author{
ANTOINE GÉRY ${ }^{1}$, ELIZABETH CHOSSON ${ }^{1}$, VIRGINIE SÉGUIN ${ }^{1}$, \\ JEAN-PHLIPPE RIOULT ${ }^{1}$, JULIE BONHOMME ${ }^{1,2} \&$ DAVID GARON $^{1}$ \\ ${ }^{1}$ ToxEMAC-ABTE, Centre F. Baclesse, Unicaen and Unirouen, Normandie University, France \\ ${ }^{2}$ Service de Microbiologie, Centre Hospitalier Universitaire de Caen, France
}

\begin{abstract}
Indoor air quality and exposure to fungal bioaerosols is a major concern in Europe where people spend most of their time indoors. 30-50\% of European homes have moisture problems facilitating mold growth, with significant health and economic consequences. Aspergilli series Versicolores are molds recurrently found in these homes which are known to cause allergies, aggravate asthma and produce sterigmatocystin, a potential human carcinogen. Recent phylogenetic studies highlighted new Versicolores species which led us to characterize fungal isolates of this section to better understand their repartition in French bioaerosols. Air samples were taken with a cyclonic biocollector indoor (hospital and mold-damaged homes) and outdoor (agricultural environment), temperature and relative humidity were recorded during sampling. Collection liquid was cultured on malt extract agar (MEA) medium supplemented with chloramphenicol $(0.05 \%, \mathrm{w} / \mathrm{v})$. Each colony was isolated and then purified on MEA. A total of 93 isolates belonging to the series Versicolores were characterized both macroscopically, microscopically and were also identified using molecular approach. We identified eight different species from bioaerosols: Aspergillus creber $(\mathrm{n}=40)$, A. jensenii $(\mathrm{n}=37)$, A. protuberus $(\mathrm{n}=6)$, A. puulaauensis $(\mathrm{n}=4)$, A sydowii $(\mathrm{n}=2)$, A. tabacinus $(\mathrm{n}=2)$, A. fructus $(\mathrm{n}=1)$, and A. amoenus $(\mathrm{n}=1)$. The phylogenetic tree performed from the BenA sequences shows homologies between isolates of the same species recovered from bioaerosols collected in the same environment which confirms the interest of Bt2a/b primers set for the identification of species of the Versicolores series. This work constitutes the first characterization of Aspergilli of the series Versicolores from French bioaerosols.
\end{abstract}

Keywords: Aspergillus, series Versicolores, bioaerosols.

\section{INTRODUCTION}

In France, as in most European countries, air quality has become a major public health issue [1]. Indeed, it is estimated that air pollution causes 48,000 deaths each year in France, which represents nearly $8 \%$ of total annual mortality between 2016 and 2019 [2]. This air quality can be altered by different factors from natural or anthropic origin [3]. Among the factors of natural origin, molds are the most frequent in indoor environments [4] where they proliferate easily in the presence of humidity, which affects 30 to $50 \%$ of European homes [5], explaining the visible presence of molds belonging mainly to the genera Aspergillus, Penicillium and Cladosporium [6] in 14-20\% of French homes [7]. These are mainly responsible for allergies [8] and sick building syndrome [9], can aggravate asthma [10] and are likely to cause infections in immunocompromised patients [11], [12]. The frequency and intensity of these symptoms are related to the level of contamination of the habitat and the duration of exposure of the population [13], which can reach up to 22 hours spent indoors per day in industrialized countries [14], [15]. However, as the relations between dampness, microbial exposure and health effects cannot be quantified precisely, no quantitative healthbased guideline values or thresholds can be recommended for acceptable levels of contamination with microorganisms [5]. Some molds are also capable of producing cytotoxic 
[16], [17] and/or genotoxic mycotoxins [18], [19]. This explains the important cost related to medical and renovation expenses in France which amount to 19 billion euros [20].

The molds of the genus Aspergillus belonging to the series Versicolores section Nidulantes are cosmopolitan. They have been found in the soil [21], in various foodstuffs for humans and animals [22], in hypersaline water [23] and in indoor environments [24]. On average, they are found in $70 \%$ of bioaerosols [6]. In addition to causing, allergies [25], aggravate asthma [26] and being linked to sick building syndrome [9], they can occasionally be responsible for onychomycosis [27] or pulmonary aspergillosis [28]. Their presence has also been shown to be an explanatory variable for various symptoms experienced by the inhabitants of dwellings damaged by moulds, such as fever, itching, headaches, dizziness or abnormally abundant expectorations [6]. Aspergilli of the series Versicolores except $A$. sydowii produce sterigmatocystin [29], a mycotoxin involved in the biosynthetic pathway of aflatoxins [30] and recognized as potentially carcinogenic to humans by IARC (group 2B) [31]. Aspergillus versicolor was considered until a few years ago as the most abundant species of the series Versicolores in bioaerosols. However, several taxonomic revisions have revealed a total of 12 new species within the series Versicolores through different phylogenetic studies. Among these, Aspergillus creber from the Latin word "creber" meaning "numerous" or "frequent" is now considered to be the most frequent species and was misidentified as Aspergillus versicolor [32], [33]. Studies on the diversity and ecology of the species of the series Versicolores confirm this hypothesis [34]. Furthermore, the different species of the series Versicolores produce different metabolites from each other and do not all have the same pathogenicity [35]-[37].

This work focuses on the molecular characterization of 93 environmental isolates of Aspergillus series Versicolores isolated from French bioaerosols in order to evaluate their diversity and to contribute to the assessment of fungal exposure to these molds.

\section{MATERIAL AND METHODS}

\subsection{Bioaerosols collection}

Bioaerosols were collected in a hospital (Centre François Baclesse, Caen, France) $(n=24)$ [38], in Serpula lacrymans- and mold-damaged homes $(n=65)$ [13], [39] and outdoor in agricultural environments (silage and hay) $(n=4)$ [40], [41].

Samples were cultured on malt extract agar (MEA) medium with $0.02 \%$ chloramphenicol (Cooper, Melun, France). Plates were incubated at $25^{\circ} \mathrm{C}$ and checked daily. Each fungal colony was isolated and purified on MEA. All Aspergillus isolates belonging to the series Versicolores $(\mathrm{n}=93)$ were stored on slant agar at $4^{\circ} \mathrm{C}$ and in a cryoprotective agent composed of sterile water (Fresenius Kabi AG, Bad Homburg, Germany) and 10\% glycerol (Carlo Erba, Val-de-Reuil, France) at $-80^{\circ} \mathrm{C}$ before molecular characterization.

\subsection{Molecular characterization}

DNA extraction was performed using a modified protocol of the Nucleospin ${ }^{\mathrm{TM}}$ Plant II kit (Macherey-Nagel, Duren, Germany). Fungal colony was introduced in a $2 \mathrm{~mL}$ microtube with glass beads. The microtube underwent two incubation cycles of $15 \mathrm{~min}$ at $80^{\circ} \mathrm{C}$ and then $15 \mathrm{~min}$ at $-80^{\circ} \mathrm{C}$. It was placed into a Qiagen Tissue Lyser with $400 \mu \mathrm{L}$ of lysis buffer PL1 (Macherey-Nagel, Duren, Germany) for $15 \mathrm{~min}$ at $20 \mathrm{~Hz}$, and incubated with $10 \mu \mathrm{L}$ of RNAse (Macherey-Nagel, Duren, Germany) and $20 \mu \mathrm{L}$ of proteinase K at $10 \mathrm{mg} / \mathrm{mL}$ (Sigma-Aldrich, St. Louis, MO, USA) at $65^{\circ} \mathrm{C}$ for $15 \mathrm{~min}$. Then, $400 \mu \mathrm{L}$ of chloroform (Sigma-Aldrich, St. 
Louis, MO, USA) was added to the mixture. The aqueous phase was recovered and extracted using the precipitation kit and washing buffer according to the protocol described by the supplier.

DNA was purified using the NucleoSpin gDNA Clean-up kit (Macherey-Nagel, Duren, Germany) following the instructions of the manufacturer. For each isolate, quantification and quality of purified DNA were realized using a NanoDrop 2000 spectrophotometer (Thermo Fisher Scientific, Waltham, MA, USA) [42].

In this study molecular characterization was performed by amplification of the betatubulin gene (BenA) using Bt2a/Bt2b (5'-GGTAACCAAATCGGTGCTGCTTTC-3'/5'ACCCTCAGTGTAGTGACCCTTGGC-3') primers [32]. Sequences obtained were compared using BLAST (Basic Local Alignment Search Tool, NCBI) to the reference sequences of the 17 Aspergillus species of the series Versicolores publicly available by Houbraken et al. [43]. Identification was considered reliable only by having a query cover $\geq 98 \%$ and a $\%$ ID $\geq 99 \%$.

Phylogenetic tree was generated using CLC Workbench 21.0.4. Tree construction was based on neighbor-joining method. Branch supports of the best maximum likelihood tree were estimated by 500 bootstrap replicates.

\section{RESULTS}

\subsection{Relative abundance in French bioaerosols}

Among all mold species recovered from bioaerosols, Aspergilli series Versicolores were the fifth most common species in bioaerosols from the hospital, the first in Serpula lacrymansand mold-damaged homes, and the third most common in agricultural environments, with concentrations ranging from $2.48 \mathrm{CFU} / \mathrm{m}^{3}$ in agricultural environments to $3.44 \times 10^{5} \mathrm{CFU} / \mathrm{m}^{3}$ in mold-damaged homes. Aspergilli series Versicolores were associated with 109, 100, and 28 other fungal species in bioaerosols collected from hospital, Serpula lacrymans- and molddamaged homes, and agricultural environments, respectively. The most represented genera in all environments were Aspergillus (A. fumigatus, A. niger, A. melleus, A. flavus, A. pseudoglaucus), Penicillium (P. chrysogenum, P. brevicompactum, P. crustosum) and Cladosporium (C. cladosporioides, C. herbarum, C. sphaerospermum) [18], [32]-[34].

As shown in Fig. 1, among all the isolates collected in French bioaerosols $(\mathrm{n}=93)$, we were able to molecularly identify eight species of the Versicolores series: Aspergillus creber $(\mathrm{n}=40)$ comes first, followed by A. jensenii $(\mathrm{n}=37)$, A. protuberus $(\mathrm{n}=6)$, A. puulaauensis $(\mathrm{n}=4)$, A. sydowii $(\mathrm{n}=2)$, A. tabacinus $(\mathrm{n}=2)$, A. amoenus $(\mathrm{n}=1)$ and A. fructus $(\mathrm{n}=1)$. Bioaerosols collected at the hospital $(\mathrm{n}=24)$ contained more $A$. jensenii $(\mathrm{n}=14)$, followed by $A$. protuberus $(\mathrm{n}=6)$, A. creber $(\mathrm{n}=3)$ and A. puulaauensis $(\mathrm{n}=1)$. Aspergillus protuberus was only found in bioaerosols collected at the hospital. Bioaerosols collected from houses damaged by Serpula lacrymans and molds $(\mathrm{n}=65)$ were mainly composed of $A$. creber $(\mathrm{n}=37)$ and A. jensenii $(\mathrm{n}=22)$. A. puulaauensis $(\mathrm{n}=3)$, A. sydowii $(\mathrm{n}=2)$, and A. amoenus $(\mathrm{n}=1)$ were also present although much less abundant. Of the four isolates obtained from bioaerosols collected from agricultural environments, we identified two isolates of $A$. tabacinus, one isolate of $A$. jensenii and one isolate of $A$. fructus.

\subsection{Phylogenetic study}

As shown in Fig. 2, the Sydowii subseries is much more represented within the bioaerosols than the Versicolores subseries. Indeed, Aspergillus creber, A. jensenii, A. puulaauensis and 
A
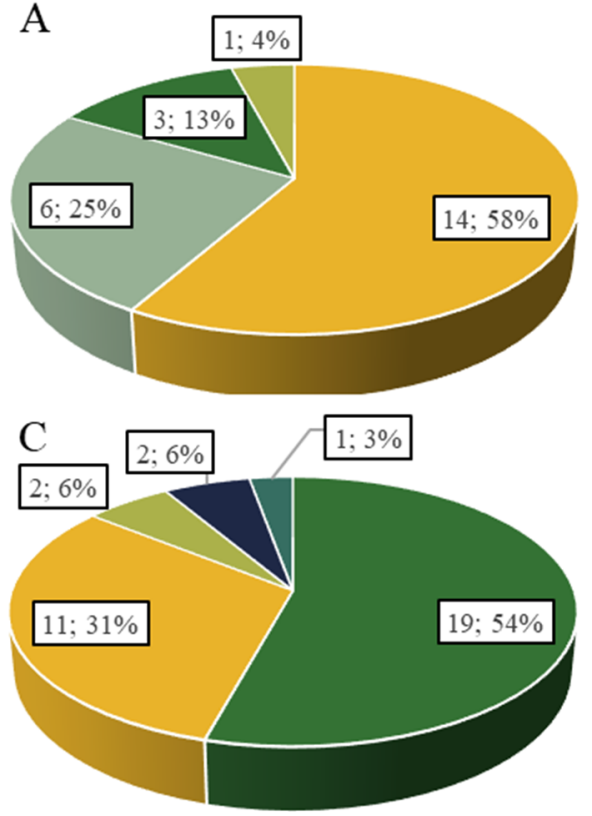

- Aspergillus creber

- Aspergillus protuberus

- Aspergillus amoenus

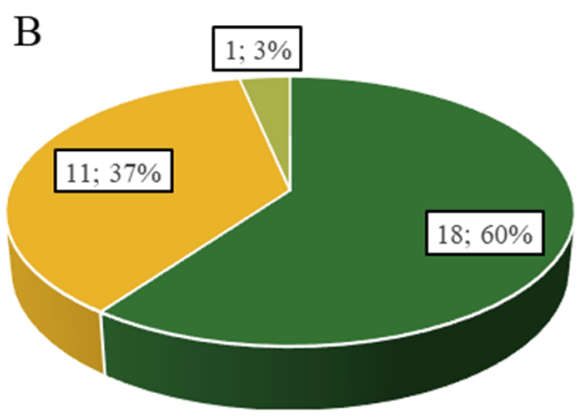

$\mathrm{D}$

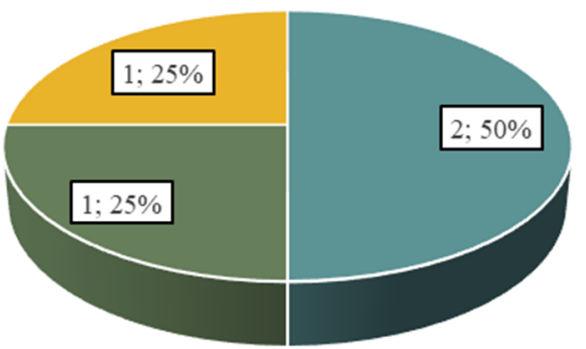

- Aspergillus sydowii

- Aspergillus tabacinus

- Aspergillus fructus

Figure 1: Relative abundances (number of isolates; percentage) of Aspergillus species belonging to the Versicolores series in bioaerosols from (A) hospital; (B) Serpula lacrymans-damaged homes; (C) mold-damaged homes; and (D) agricultural environments.

A. sydowii species represent 83 isolates (89.2\%) of the isolates found in French bioaerosols. The species belonging to the Versicolores subseries (Aspergillus protuberus, A. tabacinus, $A$. fructus and $A$. amoenus) are much less present in bioaerosols. Indeed, these represent only ten isolates (10.8\%) although Aspergillus protuberus is the third most frequently isolated species of the Versicolores series from bioaerosols. The use of the $\mathrm{Bt} 2 \mathrm{a} / \mathrm{b}$ primers set allowed the construction of a phylogenetic tree with a clear distinction of the eight species belonging to the Versicolores series found in French bioaerosols and to determine the specific richness for each environment where they were collected. In descending order, the highest species richness was found in bioaerosols from mold-damaged homes $(n=5)$, followed by those collected at the hospital $(\mathrm{n}=4)$ and tied for third place Serpula lacrymans-damaged homes and in agricultural environments $(\mathrm{n}=3)$. Aspergillus tabacinus and A. fructus were only found in bioaerosols collected from agricultural environments. Aspergillus jensenii was the only species found in all environments. We also observed a similar distribution of Versicolores species in contaminated homes regardless of whether they were contaminated by Serpula lacrymans or molds. The amplification of the BenA sequence has allowed the identification of nucleotide variations sufficient to discriminate between different strains within the same species and environment (especially for A. jensenii). 


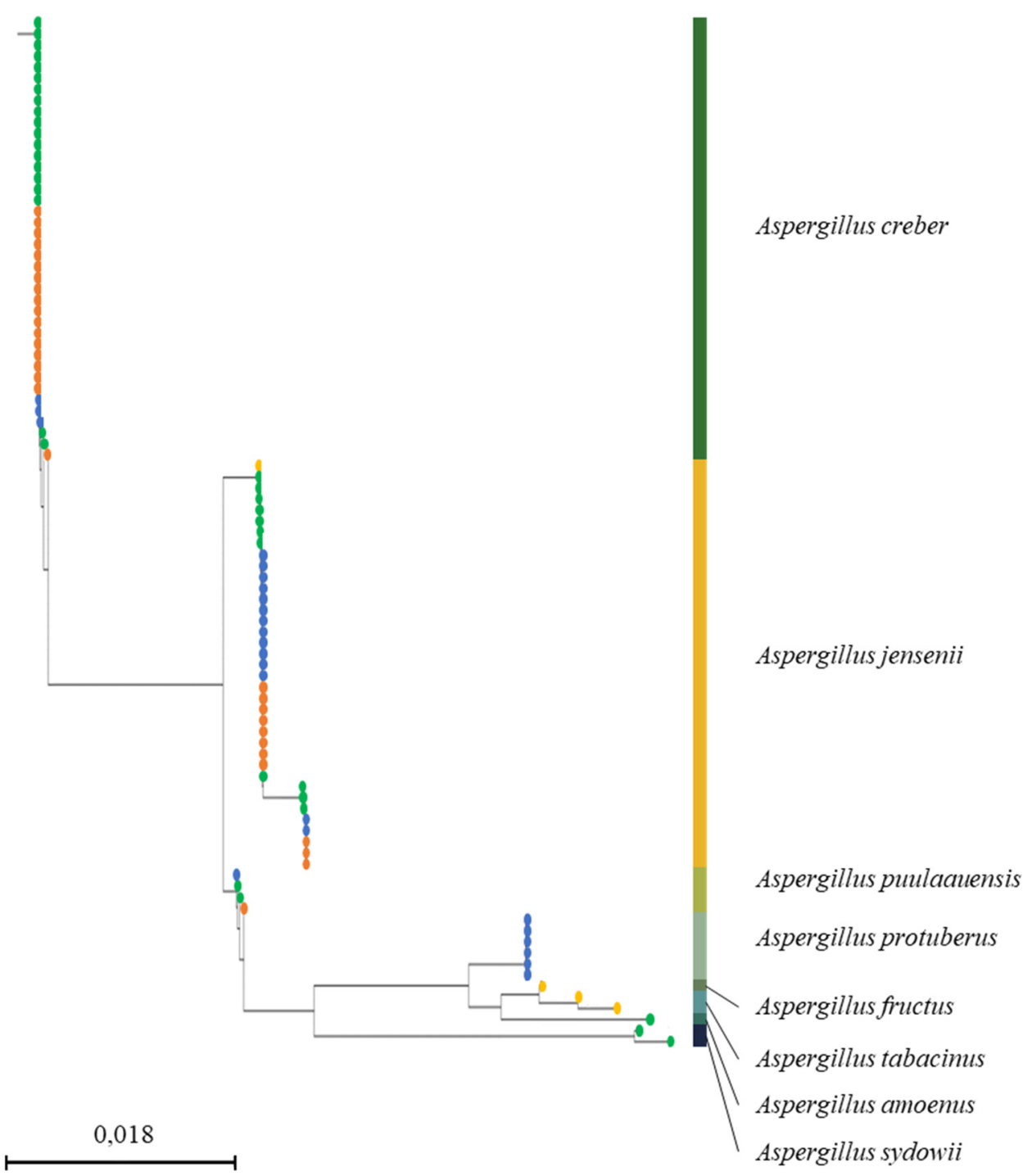

Figure 2: Phylogenetic tree of Aspergillus series Versicolores species found in French bioaerosols calculated from DNA sequence data from BenA (isolates from molddamaged homes bioaerosols in green, from Serpula lacrymans-damaged homes bioaerosols in orange, from hospital bioaerosols in blue and from agricultural environments bioaerosols in yellow).

\section{DISCUSSION}

Ninety-three fungal isolates recovered from bioaerosols collected in a hospital, in contaminated homes and in agricultural environments were molecularly identified as belonging to species of the Aspergillus Versicolores series. This study constitutes the first report on the distribution of these species in French bioaerosols molecularly characterized by ben $A$ gene amplification. We were able to group these isolates under eight different species, 
which constitutes one of the highest specific richness reported to date. Indeed, other studies conducted on isolates collected from indoor environments (air and dust samples) identified by $C a M$ gene amplification show that species richness within the Versicolores series varies from three (Aspergillus creber, A. jensenii and A. protuberus) to 11 species (A. creber, $A$. jensenii, A. puulaauensis, A. tennesseensis, A. venenatus, A. amoenus, A. fructus, $A$. griseoaurantiacus, A. pepii, A. protuberus and A. sydowii) [36], [44]. Aspergillus creber and $A$. jensenii were the most frequently isolated species from French bioaerosols $(83 \%$ of isolates), which is consistent with studies on Aspergillus species belonging to the Versicolores series that always find these species, and especially with the study by Jakšić et al. where these two species represent $62 \%$ of isolates [36], [45]. However, we did not find Aspergillus cvjetkovicii, A. griseoaurantiacus, A. pepii, A. tennesseensis, or A. venenatus, which have been isolated from indoor air bioaerosols in the United States and Croatia [33], [36], [45] but are more frequently found in various foods and feeds and in soil [36], [46]. A. protuberus and $A$. puulaauensis remain quite recurrent in indoor air and dust, as reported by Micheluz et al. [44] and Jakšić et al. [36]. Aspergillus sydowii was only isolated from two bioaerosols collected from contaminated homes, confirming its presence at a rather low frequency in air compared to food matrices or human pathology [33], [37], [46]. Aspergillus amoenus was isolated by Jurjevic et al. [33] from different sources (mammary gland, cured meat, Berberis sp. fruit) but also from indoor dust in Japan [46], which may explain why we found so few among all the collected isolates. This is the first time that Aspergillus tabacinus has been isolated from bioaerosols, whereas it is more frequently isolated from tobacco, cured meat, or plants (corn) [33]. We also found an isolate of Aspergillus fructus that had never been identified in bioaerosols, although it had been previously recovered from dust of waterdamaged and control houses in Croatia [24]. However, this species seems to be more frequently identified from fruits [33]. Aspergillus versicolor stricto sensu was not found in our bioaerosols, confirming the observations of Jurjevic et al. [33] and Kobayashi et al. [46], who reported its presence only in food products (dairy feeds, rice and noodles). Our work shows a large preponderance of species of the Sydowii subseries in indoor air bioaerosols which is in agreement with all studies that have investigated Aspergillus species of the Versicolores series in bioaerosols whether in Croatia [36], the United Kingdom [32], the USA [33], Italy [44] or Japan [46]. Although we have no other point of comparison to date and despite the small number of isolates recovered, it is interesting to note that species of the Versicolores subseries seem to be more frequent in outdoor air collected from agricultural environments. The creation of the phylogenetic tree from the BenA sequences shows homologies between isolates of the same species recovered from bioaerosols collected in the same environment which confirms the interest of the use of the $\mathrm{Bt} 2 \mathrm{a} / \mathrm{b}$ primers set for the identification of species of the Versicolores series.

[1] Anses - Agence nationale de sécurité sanitaire de l'alimentation, de l'environnement et du travail, Enjeux autour de la qualité de l'air. https://www.anses.fr/fr/content/ enjeux-autour-de-la-qualit\%C3\%A9-de-1\%E2\%80\%99air. Accessed on: 31 Aug. 2021.

[2] Medina, S. et al., Impact de la polution de l'air ambiante sur la mortalité en France métropolitaine. Direction de la communication Santé Publique France: France. Apr. p. 63. Report No: 2609-2174, 2021.

[3] Jurewicz, J., Dziewirska, E., Radwan, M. \& Hanke, W., Air pollution from natural and anthropic sources and male fertility. Reprod. Biol. Endocrinol., 16(1), p. 109, 2018. 
[4] Andersen, B., Frisvad, J.C., Søndergaard, I., Rasmussen, I.S. \& Larsen, L.S., Associations between fungal species and water-damaged building materials. Appl. Environ. Microbiol., 77(12), pp. 4180-4188, 2011.

[5] WHO Regional Office For Europe, WHO Guidelines for Indoor Air Quality: Dampness and Mould, World Health Organization: Geneva, 2009. http://www.ncbi.nlm.nih.gov/books/NBK143941/. Accessed on: 11 May 2021.

[6] Delanoë, A., Heutte, N., Gente, S., Séguin, V. \& Garon, D., Relationships between exposure to bioaerosols, moldy surface and symptoms in French mold-damaged homes. Atmosphere. 11(3), p. 223, 2020.

[7] ANSES, Moisissures dans le bâti. p. 374, Report No.: 979-10-286-0126-3, 2016.

[8] Rick, E, Woolnough, K., Pashley, C. \& Wardlaw, A., Allergic fungal airway disease. J. Investig. Allergol. Clin. Immunol., 26(6), pp. 344-354, 2016.

[9] Norbäck, D. et al., Rhinitis, ocular, throat and dermal symptoms, headache and tiredness among students in schools from Johor Bahru, Malaysia: Associations with fungal DNA and mycotoxins in classroom dust. PLOS ONE, 11(2), e0147996, 2016.

[10] Tiotiu, A.I. et al., Impact of air pollution on asthma outcomes. IJERPH, 17(17), p. $6212,2020$.

[11] Gletsou, E., Ioannou, M., Liakopoulos, V., Tsiambas, E., Ragos, V. \& Stefanidis, I., Aspergillosis in immunocompromised patients with haematological malignancies. $J$. BUON, 23(7), pp. 7-10, 2018.

[12] Spellberg, B., Edwards, J. \& Ibrahim, A., Novel perspectives on mucormycosis: Pathophysiology, presentation, and management. Clin. Microbiol. Rev., 18(3), pp. 556-569, 2005.

[13] Delanoë, A. et al., Bioaerosols exposure assessment in mold-damaged houses in Normandy, France. WIT Transactions on Ecology and the Environment, vol. 230, pp. 313-320, 2018.

[14] Boulanger, G. et al., Socio-economic costs of indoor air pollution: A tentative estimation for some pollutants of health interest in France. Environment International, 104, pp. 14-24, 2017.

[15] World Health Organization, Indoor air pollutants: Exposure and health effects. Report on a WHO meeting, Nördlingen, 8-11 June 1982. World Health Organization, Regional Office for Europe: Copenhagen, 1983. 42 pp.

[16] Bailey, B. et al., The mycotoxin deoxynivalenol significantly alters the function and metabolism of bovine kidney epithelial cells in vitro. Toxins, 11(10), p. 554, 2019.

[17] Schmutz, C., Cenk, E. \& Marko, D., The Alternaria mycotoxin alternariol triggers the immune response of IL-1 $\beta$-stimulated, differentiated caco-2 cells. Mol. Nutr. Food Res., 63(20), 1900341, 2019.

[18] Benkerroum, N., Chronic and acute toxicities of aflatoxins: Mechanisms of action. IJERPH, 17(2), p. 423, 2020.

[19] Malir, F., Ostry, V., Pfohl-Leszkowicz, A., Malir, J. \& Toman, J., Ochratoxin A: 50 years of research. Toxins, 8(7), p. 191, 2016.

[20] Kopp, P. et al., Étude exploratoire du coût socio-économique des polluants de l'air intérieur. ANSES, Report No.: 978-2-11-138465-1, 2014.

[21] Domsch, K.H., Gams, W. \& Anderson, T.H., Compendium of Soil Fungi. Academic Press: London and New York, 1980.

[22] Pitt, J.I. \& Hocking, A.D., Fungi and food spoilage, 2009. DOI: $10.1007 / 978-0-387-92207-2$. 
[23] Kis-Papo, T., Kirzhner, V., Wasser, S.P. \& Nevo, E., Evolution of genomic diversity and sex at extreme environments: Fungal life under hypersaline Dead Sea stress. Proceedings of the National Academy of Sciences, 100(25), pp. 14970-14975, 2003.

[24] Jakšić Despot, D. \& Šegvić Klarić, M., A year-round investigation of indoor airborne fungi in Croatia. Archives of Industrial Hygiene and Toxicology, 65(2), pp. 209-218, 2014.

[25] Vincent, M. et al., Investigation of inflammatory and allergic responses to common mold species: Results from in vitro experiments, from a mouse model of asthma, and from a group of asthmatic patients. Indoor Air, 27(5), pp. 933-945, 2017.

[26] Mintz-Cole, R.A., Gibson, A.M., Bass, S.A., Budelsky, A.L., Reponen, T. \& Hershey, G.K.K., Dectin-1 and IL-17A suppress murine asthma induced by Aspergillus versicolor but not Cladosporium cladosporioides due to differences in $\beta$-glucan surface exposure. JI, 189(7), pp. 3609-3617, 2012.

[27] Torres-Rodríguez, J.M., Madrenys-Brunet, N., Siddat, M., López-Jodra, O. \& Jimenez, T., Aspergillus versicolor as cause of onychomycosis: Report of 12 cases and susceptibility testing to antifungal drugs. J. Eur. Acad. Dermatol. Venereol., 11(1), pp. 25-31, 1998.

[28] Charles, M.P., Invasive pulmonary aspergillosis caused by Aspergillus versicolor in a patient on mechanical ventilation. $A M J$, pp. 632-634, 2011.

[29] Kubosaki, A. et al., A new protocol for the detection of sterigmatocystin-producing aspergillus section versicolores using a high discrimination polymerase. Biocontrol. Sci., 25(2), pp. 113-118, 2020.

[30] Schwelm, A. \& Bradshaw, R.E., Genetics of dothistromin biosynthesis of dothistroma septosporum: An update. Toxins, 2(11), pp. 2680-2698, 2010.

[31] IARC IA for R on C. Agents classified by the IARC Monographs, 56, 2012.

[32] Visagie, C.M. et al., Aspergillus, Penicillium and Talaromyces isolated from house dust samples collected around the world. Studies in Mycology, 78, pp. 63-139, 2014.

[33] Jurjevic, Z., Peterson, S.W. \& Horn, B.W., Aspergillus section Versicolores: Nine new species and multilocus DNA sequence based phylogeny. IMA Fungus, 3(1), pp. 5979, 2012.

[34] Géry, A., Rioult, J.-P., Heutte, N., Séguin, V., Bonhomme, J. \& Garon, D., First characterization and description of aspergillus series versicolores in French bioaerosols. JoF, 7(8), p. 676, 2021.

[35] Kobayashi, N. et al., Microflora of mycotoxigenic fungi in rice grains in Kyushu region of Japan and their changes during storage under non-controlled conditions. Biocontrol. Sci., 24(3), pp. 161-166, 2019.

[36] Jakšić, D., et al., Fungi and their secondary metabolites in water-damaged indoors after a major flood event in eastern Croatia. Indoor Air, 31(3), pp. 730-744, 2021.

[37] Siqueira, J.P.Z. et al., Species diversity of Aspergillus section Versicolores in clinical samples and antifungal susceptibility. Fungal Biology, 120(11), pp. 1458-1467, 2016.

[38] Heutte, N. et al., Suivi de la qualité de l'air dans un centre de lutte contre le cancer: Evaluation et caractérisation de l'exposition aux contaminants fongiques (Biohospitalair). Hygiènes, 2016. https://www.hygienes.net/boutique/hygienes2/suivi-de-qualite-de-lair-centre-de-lutte-contre-cancer-evaluation-caracterisation-delexposition-aux-contaminants-fongiques-biohospitalair/. Accessed on: 24 Jun. 2021.

[39] Pottier, D. et al., Airborne molds and mycotoxins in Serpula lacrymans-damaged homes. Atmospheric Pollution Research, 5(2), pp. 325-334, 2014. 
[40] Lanier, C., Richard, E., Heutte, N., Picquet, R., Bouchart, V. \& Garon, D., Airborne molds and mycotoxins associated with handling of corn silage and oilseed cakes in agricultural environment. Atmospheric Environment, 44(16), pp. 1980-1986, 2010.

[41] Séguin, V. et al., Effect of agricultural and environmental factors on the hay characteristics involved in equine respiratory disease. Agriculture, Ecosystems and Environment, 135(3), pp. 206-215, 2010.

[42] Desjardins, P. \& Conklin, D., NanoDrop microvolume quantitation of nucleic acids. JoVE, 1, p. 2565, 2010.

[43] Houbraken, J. et al., Classification of Aspergillus, Penicillium, Talaromyces and related genera (Eurotiales): An overview of families, genera, subgenera, sections, series and species. Studies in Mycology, 95, pp. 5-169, 2020.

[44] Micheluz, A. et al., Detection of volatile metabolites of moulds isolated from a contaminated library. Journal of Microbiological Methods, 128, pp. 34-41, 2016.

[45] Jakšić, D. et al., Species diversity and cytotoxic potency of airborne sterigmatocystinproducing Aspergilli from the section Versicolores. Sci. Total Environ., 562, pp. 296304, 2016.

[46] Kobayashi, N. et al., Distribution of sterigmatocystin-producing Aspergilli in Japan. Food Saf (Tokyo), 6(2), pp. 67-73, 2018. 